\title{
Trust transfer and the intention to use app-enabled carpooling service
}

\author{
Minhua Wu \\ Yanshan University, Qinhuangdao, China, and \\ Stern Neill \\ California Polytechnic State University, San Luis Obispo, California, USA
}

\begin{abstract}
Purpose - In China, with the rapid dissemination of mobile communications technology along with congested traffic and increasingly expensive transportation costs, consumers are turning to smartphoneenabled, ride-sharing services. Sharing economy requires trust in strangers. Based on trust transfer theory and a dyadic conceptualization of trust from cognitive to affective, the purpose of this study is to examine trust building through the use of Didi, a third-party, ride-sharing platform that mediates exchanges among strangers.

Design/methodology/approach - Structural equation modeling (SEM) results based on 242 observations indicate that the platform functions as an important enabler of trust, which influences a consumer's behavioral intention.

Findings - Specifically, Didi's reputation and security assurance have a positive influence on passengers' cognitive trust in drivers. There is also evidence that the interaction as mediated by the app between passengers and drivers helps the formation of affective trust, while the results do not support a relationship between cognitive and affective trust.

Originality/value - The research findings address trust transference between participants in the sharing economy and its effects, which have significant theoretical and practical implications and offer opportunities for future research in other sectors of the sharing economy.
\end{abstract}

Keywords Ride sharing, Trust transfer, Cognitive trust, Affective trust, Behavioral intention

Paper type Research paper

Globally, the sharing economy has motivated people to access strangers' homes and cars with the help of online services or smartphone applications such as Uber and Airbnb (Tanz, 2014). In China, with the rapid dissemination of mobile communications technology along with congested traffic and increasingly expensive transportation costs, consumers are turning to smartphone-enabled, ride-sharing services. Launched as a cab-hailing service in 2012, Didi Chuxing (hereafter Didi) acquired Uber in China, defeated rival Kuaidi Dache and gained a market share of 92.5\% in 2017 (Industry Information Network, 2017). While Didi offers a variety of services, Didi Hitch, a social ride sharing application that matches drivers and passengers who share similar routes, is a unique service offered by Didi compared to its competitor Uber. Since introduction in June 2015, Didi Hitch has built a pool of about 3m registered drivers and operated in more than 300 Chinese cities.

Didi's extraordinary growth has caught the attention of academic scholars. Data provided by Didi's online service have informed the field of urban and traffic planning (Ma and Long, 2020). Though few in quantity, research on Didi itself has also been conducted to examine service quality and user perceptions (Ma et al., 2019). But no prior research has studied the process and mechanism of Didi's mediating role in building trust between strangers as a third-party platform. Given the important role of trust in the usage of sharing services and its limited empirical scrutiny, Möhlmann (2015) calls for further investigation of the trust concept and its multifaceted characteristics in the context of collaborative consumption. Based on trust transfer theory (Stewart, 2003; Yang et al., 2015) and the dyadic conceptualization of cognitive and affective trust (McAllister, 1995), this study examines trust building through the use of Didi Hitch and its effects among car owners and passengers 
who do not have familial ties or kinship. Specifically, this research seeks to answer the following three questions in the Chinese context:

(1) How do beliefs in a third-party platform (i.e. Didi) influence trust between parties engaged in collaborative consumption (i.e. passengers and drivers)?

(2) How does interaction influence trust between parties engaged in collaborative consumption (i.e. passengers and drivers)?

(3) What effects do cognitive and affective trust have on the intention of buyers (i.e. passengers) to continue to engage in collaborative consumption?

\section{Literature review and theoretical framework}

\section{Trust in the sharing economy}

In the Internet-facilitated sharing activity, trust is built in a triadic framework with three participants: a platform enabler, a peer service provider and a customer (Kumar et al., 2017; Benoit et al., 2017). Trust is fundamental to the sharing economy, as the risks associated with participation are much higher compared to conventional, two-sided markets (Kumar et al., 2017; Aw et al., 2019), particularly in the absence of traditional factors (e.g. eye contact) or external systems (e.g. credit and monetary systems) (Molz, 2013). While Didi describes Hitch as a beautiful encounter followed by exciting experiences, the service entails risks; e.g. monetary, emotional and safety risks.

Trust in the sharing economy falls within the research field of online trust. With the allowance of interaction and collaboration among online users and the development of mobile Internet, mainstream online trust research is increasingly about the social economy (Hajli et al., 2017), followed by mobile Internet commerce and a mix of mobile and social economy (Xin et al., 2015; Chang et al., 2016). While online trust research has been extensive and informative, most prior research has taken trust as a static and unidimensional concept (Punyatoya, 2019), and the study focus either buyer-seller relationships (i.e. between app and user like in conventional two-sided markets) or the dyad relationship among app users. In observing the situation, it is necessary to adopt a more robust conceptualization of trust to examine its role in an online environment. In addition, the focus of the current study is within an app-mediated sharing economy, which imposes a new boundary for trust study based on a triad relationship among platform enabler, service provider and customer.

\section{Cognitive and affective trust}

The sharing economy demands trust in strangers. It is an essential ingredient for transactions in online peer-to-peer marketplaces since two strangers are unlikely to engage in a monetary transaction without trusting one another (Ponte et al., 2015). As trust is generally treated as a static cognitivist phenomenon in prior research (Akrout and Mbaye, 2017), this study instead applies a two-dimensional framework of trust as suggested by McAlister (1995).

McAllister (1995) identified two dimensions of trust, namely, cognitive trust and affective trust developed among managers and professionals in organizations. Cognitive trust refers to trust in evidence of peer reliability and dependability, while affective trust is based on emotional inputs and demonstrated as reciprocated care and concern between peers. He believes that affective trust develops from cognitive trust, but should be viewed as a distinctive form of interpersonal trust. As a relationship unfolds and grows, cognitive trust can be complemented by the development of affective trust as the scale and scope of interactions tends to increase over time. Akrout and Mbaye, 2017 empirically test a 
multistage trust building process that integrates calculative trust, cognitive trust, affective trust and behavioral trust in supplier-buyer relationships. Calculative, cognitive and affective trust are presented as a continuum from exploration to maintenance stages. Behavioral trust is considered a consequence of affective trust.

In online environments, scholars have called for "a break with the conventional cognitiondriven paradigm of studying user reactions to technology" (Cyr et al., 2009, p. 540). Wang et al. (2016) examined the rational and social appeals of the design of online product recommendation agents (RAs) on both cognitive and affective outcome of RA persuasion. Wang et al. (2016) addressed the affective reactions of users to technology; but without interaction between people, their study is still a static approach with no implications to the trust building process in the sharing economy.

The uniqueness of the current study is that the sharing economy requires trust in strangers with no prior knowledge and for a relationship that is more transactional in nature. As discussed above, three relationships exist - among the service provider, the service enabler and the customer in the sharing economy. The role of mobile apps in mediating exchanges among strangers (drivers and passengers) has not been examined previously. It is unclear how the trust between drivers and passengers is developed. This study seeks to address the trust formation process between drivers and passengers by examining the track record of the app on cognitive trust in drivers and the degree to which passenger-driver interaction can help the formation of affective trust in drivers. It is a story of strangers becoming friends in a somewhat transactional circumstance.

\section{Trust transfer theory}

Trust transfer refers to the cognitive process during which two unknown entities build trust because of the trustee's relationship with an entity that is known to the trustor (Stewart, 2003; Yang et al., 2015). The transference is normally from a more reputable trustee to a less wellknown entity (Doney and Cannon, 1997).

The extant research supports the trust transfer theory in three particular settings. First, trust transfer occurs within different channels of the same company. For example, trust transfer from an entity's offline channel to its online counterpart (Yang et al., 2008) or from web to mobile channels (Wang et al., 2013). Second, transference occurs between two different entities within the same channel. For example, Stewart (2003) found that consumers are directed to an unfamiliar website because of their hyperlinks shown in an established trusted website. Third, trust may also be transferred from a reputable thirdparty to a collaborative project that it sponsors or endorses. Czakon and Czernek (2016) found that local government support helped to build trust among entrepreneurs entering into network coopetition. Trust transfer is a common technique employed to establish relationships between parties that are strangers (Dong et al., 2007). Lee and Ilyoo Hong (2019) empirically examined the trust transfer path from review site to reviewer community, then to specific reviewer in consumers' online review adoption process. The research of Xiao et al. (2019) also supports the trust transfer from the intermediary platform to the user community and then to the focal merchant in online-to-offline commerce. Thus, trust transfer theory provides a suitable theoretical framework to understand the trust building process among three participators (platform, service provider and customer) in the sharing economy context.

\section{Trust studies about China}

Culture and trust are closely related (Doney and Cannon, 1997). Previous e-commerce studies find that culture plays an important role in the formation of trust (Xin et al., 2015). China has its unique cultural traits as a guanxi society rooted in Confucianism (King, 1991). Guanxi is 
defined as a dynamic process of building personal connections or relationships for the exchange of favor (Fang, 2002). Trust (xin) and feeling (qing) are the two predictors for a quality guanxi (Chen and Chen, 2004). Xin includes sincerity and ability, and qing consists of obligation and affection. A person is trustworthy when he or she is "sincere, honest, credible, reliable and capable" (Chen and Chen, 2004, p. 313). The ability-based trust corresponds with cognitive trust, the rational basis of trust. Cognitive trust derives from the trustee's integrity, expertise, competence and reliability. The qing dimension of trust corresponds with affective trust, which occurs when people put emotional feelings based on repeated social exchanges in the trust building process (Chai and Dibb, 2014). Buchan and Croson (2004) find Chinese exhibit more trusting and trustworthy behavior with partners closer to them in social distance. Further, trust and cooperation do exist among non-kin Chinese through guanxi. However, in different forms of family, helper and business guanxi, trust ranges from more affective to more calculative (Fan, 2002).

It is commonly believed that in universal cultures, like the United States, the trust building process is more calculative. While in particular societies, like China, more personal affection will be put in guanxi building (Wang, 2007). Adopting social exchange theory to compare guanxi in China and exchange relationship in the West, it can be found that guanxi approximates social exchange relationships. Social exchange relationships refer to "interpersonal connections" and involve one party supporting another to engender beneficial outcomes (Cropanzano and Mitchell, 2005). According to Blau (1964), "only social exchange tends to engender feelings of personal obligations, gratitude and trust; purely economic exchange as such does not" (p. 94). Social exchange is also guided by rule of reciprocity (Cropanzano and Mitchell, 2005) with trust as an important tenet (Park et al., 2015). But the motive for reciprocity behavior in guanxi is face saving instead of mutuality (Lee et al., 2001), which is less calculative.

\section{Conceptual model and hypothesis}

\section{Research model}

This paper examines both antecedents and effects of cognitive and affective trust to explain the trust building process within the sharing economy context. It is based on trust transfer theory (Stewart, 2003; Yang et al., 2015) and the dyadic conceptualization of cognitive and affective trust as suggested by McAllister (1995). The measurement of trust is based on a spectrum from cognitive to affective. The research framework is summarized in Figure 1, following which the theoretical support for the hypotheses is presented.

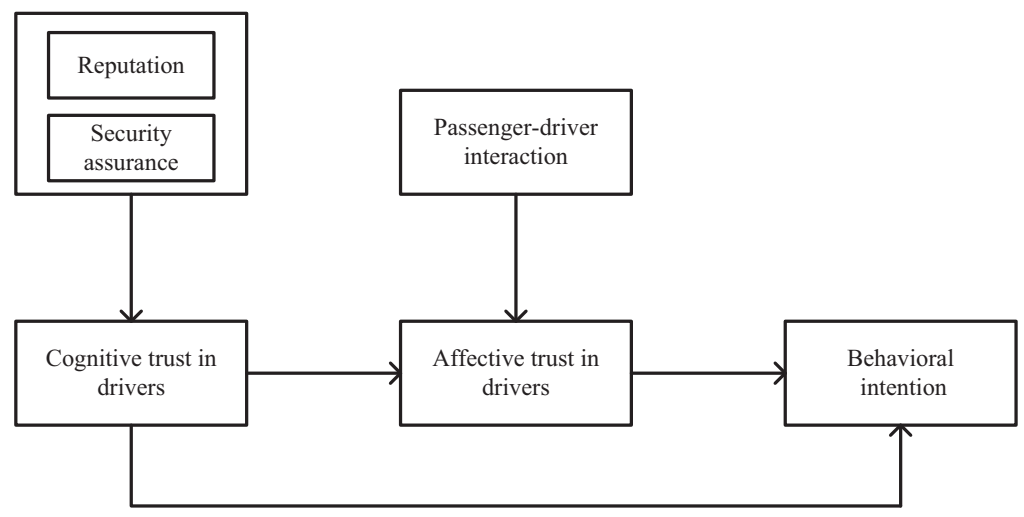

Figure 1. Conceptual model 


\section{Trust transfer within a shared economy context}

Empirical studies support the trust transfer from a reputable third-party to the collaborative project that it sponsors or endorses (Czakon and Czernek, 2016). In this research, although drivers and passengers are strangers, passengers know the Didi platform. It is commonly believed that passengers' initial trust or willingness to receive Didi drivers' service is because of Didi platform's mediation; however, no prior study has examined the process and mechanism of this mediation role. In this research, we propose that because of Didi's reputation and security assurance to protect passengers, passengers believe that Didi drivers are reliable and dependable.

Specifically, scholars have found that reputation gained from a third-party that confirms the partner's capabilities and motives helps to build trust between partners who have no previous exchange relationship (Akrout and Mbaye, 2017). Johnson and Grayson (2005) argue that when a customer is not familiar with a service provider, he/she makes the judgment about the service provider based on the reputation of the firm. A positive reputation signals lower risk and greater reliability (Akrout and Mbaye, 2017); however, scholars claim that during the trust formation phase, trust will be more cognitive and calculative (Czakon and Czernek, 2016). Thus,

H1a. The reputation of the app perceived by passengers is positively related to the passengers' cognitive trust in drivers.

In carpooling with strangers, there are risks (e.g. unsafe driving, sexual harassment, rape, robbery, etc.) that may have a negative influence on passengers' trust formation. In previous studies, structural assurance, which describes the institutional environment that supports consumer perception of safe, secure and reliable transactions (e.g. guarantees, regulations and promises), is critical in shaping initial trust in technology and protecting consumers from system-specific uncertainties and risks (McKnight et al., 2002) and is an important antecedent of trust (Srivastava et al., 2010). In this study, security assurance is used to describe the structural guarantee of Didi in protecting passengers from driver-specific uncertainties and risks and should support beliefs in trust. Therefore,

H1b. The security assurance of the carpool app is positively related to the passengers' cognitive trust in drivers.

The dyadic conceptualization of trust holds that interpersonal trust has both cognitive and affective foundations (Lewis and Wiegert, 1985), as the former represents the rational element of trust and the latter the emotional bond between persons. According to McAllister (1995), cognitive trust influences affective trust, especially in the early stage of trust formation. He explains the reason behind the relationship is that people will invest further in affective relationship only after their baseline expectations for peer reliability and dependability are met. In the online context, previous research also shows that cognitive trust is formed before affective trust and has a positive effect on affective trust formation (Akrout and Mbaye, 2017).

With the introduction of Didi Hitch in 2015, most passengers had little experience with the carpool service or its drivers. In most instances, passengers likely made the decision to try Didi Hitch because they are users of Didi Express/Premier. Using Didi was promoted to offer the opportunity of a "beautiful encounter" with strange but interesting people; however, it is uncertain that such an encounter would form a relationship. Ultimately, passengers decide whether to maintain a continuous interaction with the drivers based on some level of cognitive trust and whether this relationship will develop into a psychological attachment, as "affect influences higher stages or 'deeper' levels of trust" (Williams, 2001, p. 379).

H2. Passengers' cognitive trust in drivers is positively related to their affective trust in drivers. 
In the absence of trust, consumers tend to have less interaction with service providers and related parties (Nofer et al., 2014). Consumers develop trust from social interaction (Chang et al., 2016). Affective trust is based on positive emotions experienced during prior interactions (Johnson and Grayson, 2005). It reflects exchanges of bonds and devotion that create mutual, personal connections between the parties (Andersen and Kumar, 2006). In the case of Didi Hitch, when the passenger starts a conversation with the driver, it is a signal that he/she is open to trust in the driver and wants to either learn more about the driver or possibly pass the time. The interaction may be followed by further contact after the service is over. According to Kumar et al. (2017), in the triadic business structure of the sharing economy, interaction strength between service provider (Didi driver) and customer (Didi passenger) determines the sustainable success of the service enabler (Didi). Perceived sociability is a dimension of source credibility (Shin, 2010). Driver-passenger interaction may lead to affective trust due to this enhanced sociability. Thus,

H3. Driver-passenger interaction with the usage of carpool app will enhance passengers' affective trust in drivers.

\section{Effects of cognitive and affective trust}

While diverse risks exist in online shopping environments compared to physical stores, trust may lower the perceived risks. The influence of trust on continuous behavioral intention is significant and positive (Fang et al., 2014). Liang et al. (2018) also found that the level of consumer trust in Airbnb has a positive influence on repurchase intention. In Didi's case, at this stage of post-usage trust, passengers' cognitive and affective trust in drivers may also be transferred to continuous behavioral intention on Didi platform. Thus,

H4a. Passengers' cognitive trust in drivers is positively related to their intention to use the app.

$H 4 b$. Passengers' affective trust in drivers is positively related to their intention to use app.

\section{Methodology}

To examine the hypotheses, survey responses were collected of Didi passengers in Beijing, Shanghai, Guangzhou and Shenzhen in February 2017, which resulted in 242 valid questionnaires. To assess measurement reliability, exploratory factor analysis and scale item analyses were conducted with measures further subjected to confirmatory factor analysis. Structural equation modeling (SEM) was used to test the proposed conceptual model based on fit with the observed model, significance of path estimates (representing the study hypotheses) and explained variance of the endogenous variables.

\section{Data collection}

The instrument was a structured survey questionnaire based on established measures (see Appendix for construct items, loadings and sources), which was developed in English, translated to Chinese by an independent language expert and back-translated by a second expert (Brislin, 1980). To ensure a cross-section of Didi passengers, 588 individuals from different cities in China were contacted in February 2017 through Sojump (Wenjuanxing), a leading online platform that provides professional survey service in China (Xiao et al., 2019). In total, 242 valid surveys were completed by Didi passengers in four cities: Beijing (34\%), Guangzhou (20\%), Shanghai (28\%) and Shenzhen (18\%). Respondents were mostly young (15\% under 25 years of age, 63\% 26-35, 19\% 36-45 and 3\% 46 and older), female (58\%) and educated (4\% no college, $18 \%$ associate degree, $64 \%$ bachelor degree and $14 \%$ graduate degree). Respondents primarily used Didi within the city ( $79 \%$ intracity, $5 \%$ cross-city and 
$16 \%$ both) with different levels of usage per month (53\% 1-5 times, 33\% 6-10 times, $12 \%$ $11-15$ times, and $2 \% 16$ or more times).

\section{Results}

Measurement results

Unidimensionality was assessed based on (1) factor loadings of at least 0.50, (2) item-to-total correlations of at least 0.35 , (3) average inter-item correlations of at least 0.15 and (4) Cronbach's alpha of 0.70 . Based on this analysis, no items were removed. The measures were further subjected to confirmatory factor analysis with all items modeled as first-order factors using the covariance matrix as input. Based on this analysis, the internal consistency estimates indicated adequate support, and the goodness-of-fit results indicated that the estimated measurement model adequately represents the observed input matrix $\left(\chi^{2}=551.58\right.$ with 237 d.f.; $\mathrm{RMSEA}=0.07$; SRMR $=0.07$; $\mathrm{TLI}=0.95 ; \mathrm{CFI}=0.96$ ). To determine that each measure was empirically distinct, discriminant validity was assessed and supported in all cases (see Table 1), as the square of the parameter estimate (phi) between each pair of constructs was less than the mean of the pair's average variance extracted estimates (Fornell and Larcker, 1981). Table 1 presents the descriptive statistics, construct validity estimates and correlations among constructs.

\section{Structural model results}

To control for measurement error, each loading estimate (lambda) was fixed as the square root of the reliability estimate, and the error term (theta) was set to one minus the reliability (Hair et al., 2010). Fixing the measurement aspect prior to estimation avoids the interaction of measurement and structural models. The overall fit of the structural model was acceptable $\left(\chi^{2}=9.01\right.$ with 6 d.f.; RMSEA $=0.05$; SRMR $=0.02$; TLI $\left.=0.99 ; \mathrm{CFI}=1.00\right)$. All paths, but one, were statistically significant $(\phi<0.01)$ with standardized path estimates presented in Table 2. The structural equations accounted for sixty-five percent of the variance in cognitive

Table 1.

Descriptive statistics, construct validity estimates and correlation coefficients

\begin{tabular}{lccccccccr}
\hline Construct & Mean & SD & AVE & 1 & 2 & 3 & 4 & 5 & 6 \\
\hline Reputation & 5.72 & 0.89 & 0.48 & 0.78 & & & & & \\
Security assurance & 5.25 & 0.97 & 0.56 & 0.67 & 0.83 & & & & \\
Driver-passenger interaction & 4.44 & 1.24 & 0.42 & 0.24 & 0.32 & 0.74 & & & \\
Cognitive trust in driver & 5.54 & 0.88 & 0.65 & 0.63 & 0.66 & 0.27 & 0.88 & & \\
Affective trust in driver & 4.61 & 1.23 & 0.58 & 0.26 & 0.39 & 0.70 & 0.31 & 0.85 & \\
Behavioral intention & 5.01 & 1.13 & 0.55 & 0.37 & 0.42 & 0.50 & 0.36 & 0.57 & 0.83
\end{tabular}

Note(s): SD = Standard deviation; AVE = Average variance extracted; Reliability estimates reported on diagonal

Table 2.

Completely standardized path estimates

\begin{tabular}{lcr}
\hline Hypotheses: Path & Estimate & T-value \\
\hline Reputation $\rightarrow$ Cognitive trust in driver & 0.38 & 2.93 \\
Security assurance $\rightarrow$ Cognitive trust in driver & 0.46 & 3.63 \\
Driver-passenger interaction $\rightarrow$ Affective trust in driver & 0.88 & 12.83 \\
Cognitive trust in drivers $\rightarrow$ Affective trust in driver & 0.06 & 1.07 \\
Cognitive trust in driver $\rightarrow$ Behavior intention & 0.22 & 3.34 \\
Affective trust in driver $\rightarrow$ Behavior intention & 0.62 & 9.42 \\
Note(s): $T$-values of values of 2.33 or greater are significant at the 0.01 level &
\end{tabular}


trust in Drivers, eighty-one percent of the variance in affective trust in drivers and fifty-two percent of the variance in behavioral intention.

For empirical support of the study's hypotheses, the direction and statistical significance of each path estimate was examined. Hypothesis 1 was supported with both reputation $(\beta=0.38, p<0.01)$ and security assurance $(\beta=0.46, p<0.01)$ related to cognitive trust in drivers. Hypothesis 2 , which posited that driver-passenger interaction was related to affective trust in drivers, was also supported $(\beta=0.88, p<0.01)$ and had the strongest antecedent effect. Contrary to expectations, hypothesis 3 was not supported with no significant effect between cognitive trust in drivers and affective trust in drivers $(\beta=0.06, p>0.05)$. Finally, there was support for hypothesis 4 with both cognitive trust in drivers $(\beta=0.22, p<0.01)$ and affective trust in drivers $(\beta=0.62, p<0.01)$ related to behavioral intention. Overall, the results offer mixed support for hypotheses 1-4 and are discussed next along with implications.

\section{Discussion}

Summary of findings

Our findings confirmed third-party platforms, like Didi, empower Chinese consumers to have the autonomy and freedom to initiate and develop voluntary relationships (Chen and Chen, 2004). Based on the results, Didi's reputation and security assurance have a positive influence on passengers' cognitive trust in drivers. There is also evidence that the interaction as mediated by the app between passengers and drivers helps the formation of affective trust; however, the results do not support a relationship between cognitive and affective trust. As for the outcome of trust, both cognitive and affective trust have positive impacts on passengers' intention to use Didi with affective trust demonstrating a stronger influence on a consumer's behavioral intention.

\section{Cultural explanation of the findings}

Three features of Chinese guanxi society may explain the current findings and the contradictory results. First, Chinese guanxi society differs for in-group and out-group members, the concept that Hofstede (1980) adopted to define collectivism. In Chinese society, trust is extended to immediate family members or relatives (in-group members), which is defined as characteristic-based trust in social exchange theory (Luo, 2002). In-group members, rather than family members or relatives, gain trust based on one's past track of creditworthiness, while a first-time relationship is usually built through an intermediary that connects both parties. Without this bridge person or past track record, it will be difficult for out-group members to gain trust. The mobile-enabled app platform performs the role of the bridge in the sharing economy and helps drive the rapid growth of Didi Hitch and Xiaozhu (China's Airbnb).

Second, Chinese guanxi is based on personal connections with emotional input (Chai and Dibb, 2013). Guanxi literature explains that trust or xinren (Yen et al., 2011) is the basis of Chinese social and business relationships (Kriz and Keating, 2010). While cognitive trust is often regarded as a precursor to affective trust, it will not necessarily lead to affective trust as the results demonstrate in this research. The cultural explanation is that in Didi's case, passengers' initial trust in drivers is transferred from the platform, as both parties have little knowledge about each other, which differs from the case as discussed by McAllister (1995). In Confucian-influenced societies, emotional ties are the basis for developing interpersonal trust, which requires a mechanism to drive the interaction between the parties involved. Without peer-to-peer interaction, it is exceedingly difficult for cognitive trust that transfers from a third-party to influence affective trust. But once the guanxi is established through 
interaction, strong emotional bonds are attached to the relationship. In the current research, driver and passenger interaction has a positive impact on affective trust.

Third, Chinese guanxi is not a separate relationship. Instead, relationships are interwoven to form the guanxi network. Guanxi network serves as a mechanism for risk-reducing and lowers transaction cost as untrustworthy behavior will be punished by the whole network (Chow, 2008). Within the network, people enjoy low uncertainty avoidance, tend to trust each other and make repeated business transactions, which have great significance for the platform's intention to build trust and form a guanxi network or virtual community in the online environment among its users.

\section{Theoretical contributions}

The present research makes several theoretical contributions. This study is the first to examine trust transfer mechanisms among participates in the sharing economy. Extant marketing research on "the nature of trust and its role" was conducted before the emergence of the sharing economy (Eckhardt et al., 2019), while this research establishes a conceptual model with three participators of the sharing economy and empirically examines how initial cognitive trust between provider and consumer is mediated by a third-party platform. The results confirm the role of service enabler in the formation of trust between drivers and passengers, who are strangers and do not have familial ties or kinship. As all information prior to the ride is from the app, the platform performs the role of a bridge to transfer passengers' attitudes and beliefs to cognitive trust in car owners.

This research also enriches the trust transfer theory in that it reveals the expansion of trust transfer settings in an online context. It shows that trust transfer occurs not only in the three particular settings as discussed above but also from a well-known third-party to affiliated individuals. The expansion has significance for researchers to further test the boundary of trust transfer settings in the sharing economy, which will have great impact on a platform's business strategy development. For example, will consumer's affective trust in service providers be transferred back to a platform? The answer to the question will help a platform in deciding its commitment in building cognitive or affective trust.

Second, this study first reveals that cognitive trust may not necessarily influence affective trust in the context of the sharing economy. In addition to the reasons stated in the cultural explanation of the findings (above), this contradictory result is also supported by the theory of perceived entitativity, which evaluates the bonded relationship between a source object and a target object in the trust transfer process (Lickel et al., 2000). According to Gong et al. (2019), perceived entitativity in terms of external bonded tie performs a more significant role in the process of affective trust transfer. But unfortunately, there is no such bonded tie existing between Didi and drivers, as the major role of Didi is a platform that mediates service offer, and drivers are not formally affiliated with the platform. Yang et al. (2019) has similar results showing that consumers' cognitive trust in a platform (Airbnb) does not mean that they are likely to be emotionally attached to actual service providers (Airbnb hosts). As is often the case in the sharing economy, affective trust can be influenced by enhancing the interaction between service providers and customers but not likely gained through the platform.

Third, the current study is the first to examine the two dimensions of trust and their respective impacts on passengers' behavioral intention in the sharing economy. The results show that both cognitive and affective trusts have a positive and independent relationship on the continuous usage intention of Didi, though affective trust has a stronger impact. It further supports that cognitive and affective trust are two empirically distinct constructs and the importance of cognitive and affective trust in building customer loyalty as demonstrated in other online contexts (Punyatoya, 2019). Since affective trust has a stronger impact on 
consumer's behavioral intention, it is suggested to encourage interaction between customers and service providers and turn them into loyal supporters and promoters of the platform.

\section{Managerial implications}

This study has significant implications for practitioners as well. First, this study informs platforms within the sharing economy of the importance to build reputation and offer protection to participants through careful design of business processes. The research results show that Didi's reputation and security assurance serve as the starting point of trust building. With the increased adoption of sharing service as well as the increased reports of extreme incidents, the role of trust might change from convincing consumers to use the service to mediating between satisfaction and repurchase intention like in the case of Airbnb (Liang et al., 2018), which is of great importance to the sustainability of the sharing economy.

Möhlmann (2015, p. 201) states that "[m]anagers need to make sure that trust building measures are implemented and communicated to respective stakeholders." Leading platforms enjoy the advantage of quickly building reputation through the agglomeration effect of good word of mouth; however, this same amplification is true when negative events occur. It is recommended that sharing economy platforms invest time, effort and money in the design of the process to offer security guarantees to both service providers and consumers. In addition, the education and communication of security assurance measures are equally important. Didi Hitch had its business suspended after two vicious incidents occurred in 2018. It went back to test the market in November 2019 when new safety measures were added and user training required to check the safety function and learn related knowledge to avoid extreme incidents.

Second, difficulties in establishing trust in strangers in the sharing economy might be of competitive advantage for non-sharing service providers who have established a reputation in certain industries (Möhlmann, 2015). The non-sharing business can leverage the reputation built in the past in the introduction of collaborative consumption. For example, in the United States, Google introduced Waze Rider, which is similar to Didi Hitch and competes with Uber as a riding service provider. The trust transfer process also helps those who are already in the sharing economy to introduce new lines of business like in the case of Didi Hitch, which was introduced after Didi Taxi, Express and Premier were successful adopted.

Third, this study provides direction for platforms to encourage interaction between service providers and receivers. Debate continues as to whether a platform should be built as a pure bridge between sharing economy participants or as a virtual community with social function. In addressing this debate, there are two questions to consider. First, would participants like to Interact with each other? And second, what will be the impact of their socialization? According to Belk (2014) and Habibi et al. (2016), the answer to the first question depends on the practice's location along the continuum of pure sharing to pure exchange, where participants move from seeking social utility to exchange utility. Most practices in the sharing economy are a kind of mix or positioned somewhere in between. As in the case of Airbnb and UberX, although economic motive was the primary driver, there are also sharing contexts that are based on social bonds (Habibi et al., 2016). Social exchange does help the formation of affective trust, which is more stable and stronger (Akrout and Mbaye, 2017) and has a greater impact on customers' intention to use the service in practices and cultures that stress emotional input. Airbnb promotes the formation of social relationships through Facebook and offers privileges to those who have social contact. Similarly, virtual communities can be established among Didi users according to the commuting route. According to social exchange theory, once the virtual community is formed, it will create "a feeling of shared binding as well as shared ethical and moral habits", which gives community members a sense that they can trust each other (Luo, 2002). That is, the community itself will perform the role of risk-reducer. 
Fourth, service enablers should realize that the closeness of their relationship with service providers determines the quality of trust transfer, which in turn will influence consumers' intention to use the service. Continuous reminders regarding safety and other issues should be done through the platform. New drivers should be trained to learn the motives and concerns of potential customers. Other innovative measures that strengthen the affiliation between service enablers and service providers should be initiated to build service providers into strategic partners of the platform. These measures will not only make service enablers and providers bonded but also will upgrade the service quality provided to customers.

\section{Limitations and future research}

Notwithstanding the contributions of this research, it has several limitations. First, although the cornerstone of trust building in the case of Didi Hitch is its reputation and security assurance, the paper does not explore how the platform can build reputation and security assurance and its impact in terms of benefits as well as costs. Second, future research should attend to possible measurement and study design issues. While study measures appear robust, variance extracted estimates for reputation and driver-passenger interaction indicate more error remains in the items than is explained by the factor structure, which future research may wish to consider. In terms of study design, the research is done with one carpooling app in China's first tier cities of Beijing, Shanghai, Guangzhou and Shenzhen. Subsequent research should be conducted in more cities to test the results. Third, this research is conducted in China, which is a collective society with unique guanxi culture. As discussed before, the trust building process in Didi may not apply in individual cultures. Further, China's younger generation, riding the wave of globalization, are increasingly individualistic and encouraged to trust in strangers (Ni and Ishii, 2019). Thus, comparative studies in other cultures and longitudinal investigation in China will be informative and amplify the trust research in the sharing economy.

\section{References}

Akrout, H. and Mbaye, F.D. (2017), "Fundamental transformations of trust and its drivers: a multistage approach to business-to-business relationships", Industrial Marketing Management, Vol. 66, pp. 159-171.

Andersen, P.H. and Kumar, R. (2006), "Emotions, trust and relationship development in business relationships: a conceptual model for buyer-seller dyads", Industrial Marketing Management, Vol. 35 No. 4, pp. 522-535.

Aw, C.X., Basha, N.K., Ng, S.I. and Sambasivan, M. (2019), "To grab or not to grab? The role of trust and perceived value in on-demand ridesharing services", Asia Pacific Journal of Marketing and Logistics, Vol. 31 No. 5, pp. 1442-1465.

Belk, R. (2014), "You are what you can access: sharing and collaborative consumption online", Journal of Business Research, Vol. 67 No. 8, pp. 1595-1600.

Benoit, S., Baker, T.L., Bolton, R.N., Gruber, T. and Kandampully, J. (2017), "A triadic framework for collaborative consumption (cc): motives, activities and resources and capabilities of actors", Journal of Business Research, Vol. 79, pp. 219-227.

Blau, P.M. (1964), Exchange and Power in Social Life, John Wiley, New York, NY.

Brislin, R.W. (1980), "Translation and content analysis of oral and written material", in Triandis, H.C. and Berry, J.W. (Ed.), Handbook of Cross-Cultural Psychology: Methodology, Allyn and Bacon, Boston, pp. 389-444.

Buchan, N. and Croson, R. (2004), "The boundaries of trust: own and others' actions in the US and China", Journal of Economic Behavior and Organization, Vol. 55 No. 4, pp. 485-504. 
Chai, J.Y. and Dibb, S. (2014), "How consumer acculturation influences interpersonal trust", Journal of Marketing Management, Vol. 30 Nos 1/2, pp. 60-89.

Chang, S.E., Shen, W.C. and Liu, A.Y. (2016), "Why mobile users trust smartphone social networking services? A PLS-SEM approach”, Journal of Business Research, Vol. 69 No. 11, pp. 4890-4895.

Chen, X.P. and Chen, C.C. (2004), "On the intricacies of the Chinese Guanxi: a process model of Guanxi development”, Asia Pacific Journal of Management, Vol. 21 No. 3, pp. 305-324.

Chow, H.S. (2008), "How trust reduces transaction costs and enhances performance in China's businesses", SAM Advanced Management Journal, (07497075), Vol. 73 No. 2, pp. 25-35.

Cropanzano, R. and Mitchell, M.S. (2005), "Social exchange theory: an interdisciplinary review", Journal of Management, Vol. 31 No. 6, pp. 874-900.

Cyr, D., Head, M., Larios, H. and Pan, B. (2009), "Exploring human images in website design: a multimethod approach”, MIS Quarterly, Vol. 33 No. 3, pp. 539-566.

Czakon, W. and Czernek, K. (2016), "The role of trust-building mechanisms in entering into network coopetition: the case of tourism networks in Poland", Industrial Marketing Management, Vol. 57 August, pp. 64-74.

Doney, P.M. and Cannon, J.P. (1997), "An examination of the nature of trust in buyer-seller relationships", Journal of Marketing, Vol. 61 April, pp. 35-51.

Dong, C.Y., Russello, G. and Dulay, N. (2007), "Trust transfer in distributed systems", IFIP International Federation for Information Processing, Vol. 238, pp. 17-29.

Eckhardt, G.M., Houston, M.B., Jiang, B., Lamberton, C., Rindfleisch, A. and Zervas, G. (2019), "Marketing in the sharing economy", Journal of Marketing, Vol. 83 No. 5, pp. 5-27.

Fan, Y. (2002), "Questioning guanxi: definition, classification and implications", International Business Review, Vol. 11 No. 5, pp. 543-561.

Fang, Y., Qureshi, I., Sun, H., McCole, P., Ramsey, E. and Lim, K.H. (2014), "Trust, satisfaction, and online repurchase intention: the moderating role of perceived effectiveness of e-commerce institutional mechanisms", MIS Quarterly, Vol. 38 No. 2, pp. 407-427.

Fornell, C. and Larcker, D.F. (1981), "Evaluating structural equation models with unobservable variables and measurement error", Journal of Marketing Research, Vol. 18 No. 1, pp. 39-50.

Gong, X., Zhang, Z.K., Chen, C., Cheung, M.K. and Lee, K.O. (2019), "What drives trust transfer from web to mobile payment services? The dual effects of perceived entitativity", Information and Management, Vol. 57 No. 7.

Habibi, M.R., Kim, A. and Laroche, M. (2016), "From sharing to exchange: an extended framework of dual modes of collaborative nonownership consumption", Journal of the Association for Consumer Research, Vol. 1 No. 2, pp. 277-294.

Hair, J.F., Black, W.C., Babin, B.J. and Anderson, R.E. (2010), Multivariate Data Analysis, Prentice Hall, Upper Saddle River, NJ.

Hajli, N., Sims, J., ArashZadeh, H.A.H., Richard, M.O. and Woodside, A.G. (2017), "A social commerce investigation of the role of trust in a social networking site on purchase intentions", Journal of Business Research, Vol. 71, pp. 133-141.

Hamari, J., Sjoklint, M. and Ukkonen, A. (2016), "The sharing economy: why people participate in collaborative consumption", Journal of the Association for information Science and Technology, Vol. 67 No. 9, pp. 2047-2059.

Hofstede, G.H. (1980), Culture's Consequences: International Differences in Work-Related Values, Sage Publications.

Industry Information Network (2017), "Study on the competitive situation of China's app-enabled transportation market 2017", November 21, available at: https://www.chyxx.com/industry/ 201711/584806.html in Chinese. 
Jin, Y., Gu, Z., Gu, J. and Zhao, H. (2007), A New Reputation-Based Trust Management Mechanism against False Feedbacks in Peer-To-Peer Systems, Web Information Systems EngineeringWISE, Springer Berlin Heidelberg, pp. 62-73.

Johnson, D. and Grayson, K. (2005), "Cognitive and affective trust in service relationships", Journal of Business Research, Vol. 58 No. 4, pp. 500-507.

King, A.Y. (1991), "Kuan-hsi network building: a sociological interpretation", Daedalus, Vol. 120 No. 20, pp. 63-84.

Kriz, A. and Keating, B. (2010), "Lessons from China about deep trust”, Asia Pacific Business Review, Vol. 16 No. 3, pp. 299-318.

Kumar, V., Lahiri, A. and Dogan, O.B. (2017), "A strategic framework for a profitable business model in the sharing economy", Industrial Marketing Management, Vol. 69, pp. 147-160.

Lee, J. and Ilyoo Hong, B. (2019), "Consumer's electronic word-of-mouth adoption: the trust transfer perspective", International Journal of Electronic Commerce, Vol. 23 No. 4, pp. 595-627.

Lee, D.J., Pae, J.H. and Wong, Y.H. (2001), “A model of close business relationship in China (guanxi)”, European Journal of Marketing, Vol. 35 Nos 1/2, pp. 51-69.

Lewis, J.D. and Weigert, A. (1985), "Trust as a social reality", Social Force, Vol. 63, pp. 967-985.

Liang, L.J., Choi, H.C. and Joppe, M. (2018), "Exploring the relationship between satisfaction, trust and switching intention, repurchase intention in the context of Airbnb", International Journal of Hospitality Management, Vol. 69, pp. 41-48.

Lickel, B., Hamilton, D.L., Wieczorkowska, G., Lewis, A., Sherman, S.J. and Uhles, A.N. (2000), "Varieties of groups and the perception of group entitativity", Journal of Personality and Social Psychology, Vol. 78 No. 2, p. 223.

Luo, X. (2002), "Trust production and privacy concerns on the Internet: a framework based on relationship marketing and social exchange theory", Industrial Marketing Management, Vol. 31, pp. 111-118.

Ma, S. and Long, Y. (2020), "Functional urban area delineations of cities on the Chinese mainland using massive Didi ride-hailing records", Cities, Vol. 97 February, pp. 1-13.

Ma, L., Zhang, X., Ding, X. and Wang, G. (2019), "Risk perception and intention to discontinue use of ride-hailing services in China: taking the example of DiDi Chuxing", Transportation Research Part F, Vol. 66, pp. 459-470.

McAllister, D.J. (1995), "Affect- and cognition-based trust as foundations for interpersonal cooperation in organizations", Academy of Management Journal, Vol. 38, pp. 24-59.

McKnight, D.H., Choudhury, V. and Kacmar, C. (2002), "Developing and validating trust measures for e-commerce: an integrative typology", Information Systems Research, Vol. 13 No. 3, pp. 334-359.

Möhlmann, M. (2015), "Collaborative consumption: determinants of satisfaction and the likelihood of using a sharing economy option again", Journal of Consumer Behaviour, Vol. 14 No. 3, pp. 193-207.

Molz, J.G. (2013), "Social networking technologies and the moral economy of alternative tourism: the case of couchsurfing.org", Annals of Tourism Research, Vol. 43 No. 7, pp. 210-230.

$\mathrm{Ni}$, S. and Ishii, K. (2019), "The influence of shopping on subjective well-being in China", Asia Pacific Journal of Marketing and Logistics, Vol. 31 No. 1, pp. 184-201.

Nofer, M., Hinz, O., Muntermann, J. and Roßnagel, H. (2014), "The economic impact of privacy violations and security breaches", Business and Information Systems Engineering, Vol. 56 No. 6, pp. 369-380.

Park, J.G., Lee, H. and Lee, J. (2015), “Applying social exchange theory in IT service relationships: exploring roles of exchange characteristics in knowledge sharing", Information Technology Management, Vol. 16, pp. 193-206.

Ponte, E.B., Carvajal-trujillo, E. and Escobarrodrıguez, T. (2015), "Influence of trust and perceived value on the intention to purchase travel online: integrating the effects of assurance on trust antecedents", Tourism Management, Vol. 47 No. 47, pp. 286-302. 
Punyatoya, P. (2019), "Effects of cognitive and affective trust on online customer behavior", Marketing Intelligence and Planning, Vol. 37 No. 1, pp. 80-96.

Shin, D.H. (2010), "Modeling the interaction of users and mobile payment system: conceptual framework", International Journal of Human-Computer Interaction, Vol. 26 No. 10, pp. 917-940.

Srivastava, S.C., Chandra, S. and Theng, Y.L. (2010), "Evaluating the role of trust in consumer adoption of mobile payment systems: an empirical analysis", Communications of the Association for Information Systems, Vol. 27 No. 29, p. 561.

Stewart, K.J. (2003), "Trust transfer on the world wide web", Organization Science, Vol. 14 No. 1 , pp. 5-17.

Tanz, J. (2014), "How Airbnb and Lyft finally got Americans to trust each other", January 29, available at: http://www.wired.com/2014/04/trust-in-the-share-economy/.

Wang, C.L. (2007), “Guanxi vs. Relationship marketing, exploring underlying differences”, Industrial Marketing Management, Vol. 36 No. 1, pp. 81-86.

Wang, N., Shen, X.L. and Sun, Y.Q. (2013), "Transition of electronic word-of- mouth services from web to mobile context: a trust transfer perspective", Decision Support Systems, Vol. 54 No. 3, pp. 1394-1403.

Wang, W., Qiu, L., Kim, D. and Benbasat, I. (2016), "Effects of rational and social appeals of online recommendation agents on cognition- and affect-based trust", Decision Support Systems, Vol. 86, pp. 48-60.

Williams, M. (2001), "In whom we trust: group membership as an affective context for trust development", Academy of Management Review, Vol. 26 No. 3, pp. 377-396.

Xiao, L., Zhang, Y. and Fu, B. (2019), "Exploring the moderators and causal process of trust transfer in online-to-offline commerce", Journal of Business Research, Vol. 98, pp. 214-226.

Xin, H., Techatassanasoontorn, A.A. and Tan, F.B. (2015), "Antecedents of consumer trust in mobile payment adoption”, Journal of Computer Information Systems, Vol. 55 No. 4, pp. 1-10.

Yang, Q., Huang, L. and Xu, Y. (2008), "Role of trust transfer in E-commerce acceptance", Tsinghua Science and Technology (English Edition), Vol. 13 No. 3, pp. 279-286.

Yang, S., Chen, Y. and Wei, J. (2015), 'Understanding consumers' web-mobile shopping extension behavior: a trust transfer perspective”, Journal of Computer Information Systems, Vol. 55 No. 2, pp. 78-87.

Yang, S.B., Lee, K., Lee, H. and Koo, C. (2019), "In Airbnb we trust: understanding consumers' trustattachment building mechanisms in the sharing economy", International Journal of Hospitality Management, Vol. 83, pp. 198-209.

Yen, D.A., Barnes, B.R. and Wang, C.L. (2011), "The measurement of guanxi: introducing the grx scale", Industrial Marketing Management, Vol. 40 No. 1, pp. 97-108.

Zeithaml, V.A., Berry, L.L. and Parasuraman, A. (1996), "The behavioral consequences of service quality", Journal of Marketing, Vol. 60 No. 2, pp. 31-46.

\section{Further reading}

Medrano, J.D. (2009), "Interpersonal trust", available at http://www.jdsurvey.net/jds/ jdsurveyActualidad.jsp?Idioma $=I \&$ SeccionTexto $=0404 \& \mathrm{NOID}=104$. 


\section{Appendix}

Construct items

Loading

Reputation Jin et al. (2007)

Didi is a market leader in China's carpooling market $\quad 0.69$

$\begin{array}{lr}\text { Didi is well-known } & 0.63\end{array}$

$\begin{array}{lr}\text { Didi has a good reputation } & 0.79\end{array}$

$\begin{array}{ll}\text { Didi has the largest user pool among similar apps } & 0.64\end{array}$

Security assurance Xin et al. (2015)

The driver verification system of Didi makes sure that the drivers are qualified 0.72

Didi's channel for passengers to give feedback about the drivers' misbehavior works well $\quad 0.74$

I am satisfied with the insurance offered by Didi, which is covered by China Ping An Insurance 0.72

$\begin{array}{ll}\text { Didi Hitch has taken proper measures to protect passengers' safety } & 0.80\end{array}$

Driver-passenger interaction McAllister (1995)

$\begin{array}{ll}\text { I often chat with the driver and/or other passengers while carpooling } & 0.61\end{array}$

$\begin{array}{ll}\text { I contact Didi drivers even when they are out of carpool service } & 0.58\end{array}$

I have found the drivers that have similar route with me through "Fellow Traveler" function $\quad 0.68$

I follow some drivers via the app

Cognitive trust in drivers Wang et al. (2016)

$\begin{array}{ll}\text { Didi drivers are well qualified and their services good } & 0.77\end{array}$

Generally speaking, Didi Hitch drivers are sincere, friendly and considerate $\quad 0.75$

$\begin{array}{ll}\text { Overall, Didi Hitch drivers are trustworthy } & 0.89\end{array}$

Overall, Didi Hitch drivers have good track record in providing the service 0.80

Affective trust in drivers Wang et al. (2016), McAllister (1995)

It's fascinating to meet both new and old friends in using Didi $\quad 0.77$

$\begin{array}{lr}\text { I enjoy the chats during carpool } & 0.81\end{array}$

$\begin{array}{ll}\text { Didi Hitch drivers are just like my friends } & 0.78\end{array}$

I would miss the time with earlier Didi drivers if I had to change the route 0.68

Behavior intention Hamari et al. (2016), Zeithaml et al. (1996)

$\begin{array}{ll}\text { I intend to use Didi Hitch in the foreseeable future } & 0.61\end{array}$

Table A1. I consider Didi Hitch my first choice for ride service 0.83

Construct items, $\quad$ I intend to increase my use of Didi Hitch in the foreseeable future 0.80

loadings and sources I will do business with Didi Hitch if its prices increase somewhat 0.70

\section{Corresponding author}

Minhua Wu can be contacted at:mhwu@ysu.edu.cn 